\title{
Two-Page Book Embeddings of 4-Planar Graphs*
}

\author{
Michael A. Bekos ${ }^{1}$, Martin Gronemann ${ }^{2}$, and \\ Chrysanthi N. Raftopoulou ${ }^{3}$
}

1 Wilhelm-Schickhard-Institut für Informatik, Universität Tübingen, Germany bekos@informatik. uni-tuebingen.de

2 Institut für Informatik, Universität zu Köln, Germany

gronemann@informatik. uni-koeln.de

3 School of Applied Mathematical \& Physical Sciences, NTUA, Greece

crisraft@mail.ntua.gr

\begin{abstract}
Back in the eighties, Heath [7] showed that every 3-planar graph is subhamiltonian and asked whether this result can be extended to a class of graphs of degree greater than three. In this paper we affirmatively answer this question for the class of 4-planar graphs. Our contribution consists of two algorithms: The first one is limited to triconnected graphs, but runs in linear time and uses existing methods for computing hamiltonian cycles in planar graphs. The second one, which solves the general case of the problem, is a quadratic-time algorithm based on the book embedding viewpoint of the problem.
\end{abstract}

1998 ACM Subject Classification G.2.2 Graph Theory

Keywords and phrases Book Embedding, Subhamiltonicity, 4-Planar Graphs

Digital Object Identifier 10.4230/LIPIcs.STACS.2014.137

\section{Introduction}

Book embeddings have a long history and arise in various application areas such as VLSI design [5]. In a book embedding the placement of nodes is restricted to a line, the spine of the book. The edges are assigned to different pages of the book. A page can be thought of as a half-plane bounded by the spine where the edges are drawn as circular arcs between their endpoints. We say that a graph admits a $k$-page book embedding if one can assign the edges to $k$ pages and there exists a linear ordering of the nodes on the spine such that no two edges of the same page cross. The minimum number of pages required to construct such an embedding is the book thickness or page number of a graph and has received much attention in the past. Yannakakis [14] describes a linear-time algorithm to embed every planar graph into a book of four pages. Bernhart et al. [2] show that a graph is two-page embeddable iff it is subhamiltonian. A subhamiltonian graph is a subgraph of a planar hamiltonian graph. It is $N P$-complete to determine whether a graph is subhamiltonian [13]. Often referred to as augmented hamiltonian cycle, a subhamiltonian cycle is a cyclic sequence of nodes in a graph that would form a hamiltonian cycle when adding the missing edges without destroying planarity. The relation between subhamiltonian cycles and two-page book embeddings is

\footnotetext{
* Work on this problem began at Dagstuhl Seminar 13151. We thank the organizers, participants and Prof. Dr. M. Kaufmann. The work of M.A. Bekos is implemented within the framework of the Action "Supporting Postdoctoral Researchers" of the Operational Program "Education and Lifelong Learning" (Action's Beneficiary: General Secretariat for Research and Technology), and is co-financed by the European Social Fund (ESF) and the Greek State.
}

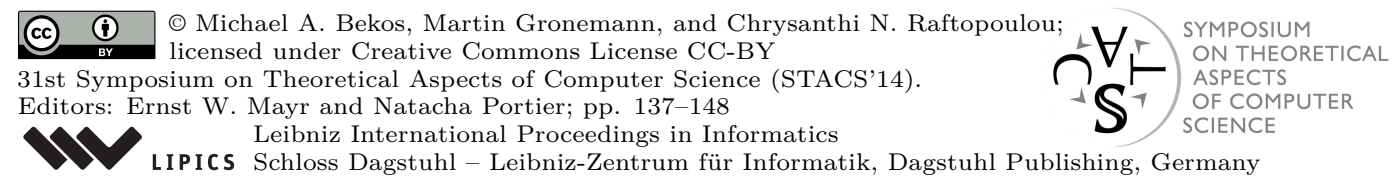




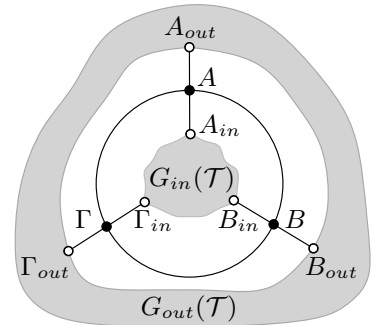

Figure 1 Triangle $\mathcal{T}$ separating $G_{\text {in }}(\mathcal{T})$ and $G_{\text {out }}(\mathcal{T})$ on removal.

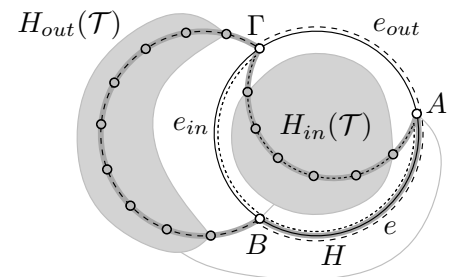

Figure 2 Merging $H_{i n}(\mathcal{T})$ (dotted) and $H_{\text {out }}(\mathcal{T})$ (dashed) into $H$ (bold gray).

quite intuitive. The order of the nodes on the spine is equivalent to the cyclic order of the subhamiltonian cycle. The edges are partitioned by whether they lie in the interior of the cycle or not.

An early result is due to Whitney [12], who proves that every maximal planar graph with no separating triangles is hamiltonian. Tutte [11] shows that every 4-connected planar graph has a hamiltonian cycle. Chiba et al. [4] provide a linear-time algorithm to find a hamiltonian cycle in a 4-connected planar graph. Chen [3] gives a proof that every maximal planar graph with at least five vertices and no separating triangles is 4-connected. Sanders [10] generalizes a theorem of Thomassen and shows that any 4-connected planar graph has a hamiltonian cycle that contains two arbitrarily chosen edges of the graph. Based on Whitney's theorem, Kainen et al. [9] show that every planar graph with no separating triangles is subhamiltonian. Another result is by Chen [3] who shows that if a maximal planar graph contains only one such triangle, then it is hamiltonian. Helden [8] improves this result further to two triangles. The aforementioned results are all related to the problem of embedding planar graphs into two pages. However, there is an extensive amount of literature on embedding various types of graphs into books; see e.g. [6]. One result that is interesting in our context is that of Heath [7], who describes a linear-time algorithm to embed any 3-planar graph into two pages.

We study the problem of embedding 4-planar graphs into books with two pages. We tackle this problem from two sides. The first approach is restricted to triconnected graphs (Section 2) but builds on existent results and is therefore of a simple nature compared to the second approach. Extending it to biconnected graphs is not straightforward, though. The algorithm of Section 3 -which is less efficient in terms of time complexity- exploits the degree restriction to construct a two-page book embedding. Due to space constraints, some of our proofs are only sketched, omitted details are given in [1].

\section{Subhamiltonicity of Triconnected 4-Planar Graphs}

In this section, we first investigate properties of separating triangles in 4-planar graphs and then we use those to derive a solution for a single separating triangle. Unlike Chen [3] and Helden [8], we are able to extend our approach to an unbounded number of triangles by exploiting the degree restriction. We say a subhamiltonian cycle $H$ crosses a face if there are two consecutive vertices in $H$ that are incident to the face but not adjacent to each other.

- Lemma 1. Every triconnected planar graph with no separating triangles has a subhamiltonian cycle that crosses every face at most once and it can be computed in linear time.

Proof. In the triconnected case, Kainen et al. [9] construct a maximal planar graph $G^{\prime}=$ $\left(V^{\prime}, E^{\prime}\right)$ by inserting a vertex into each non-triangular face of $G$ and connect it to the vertices 
of that face. Clearly, this takes linear time and $G^{\prime}$ is 4-connected. We can use the linear-time algorithm of Chiba et al. [4] to obtain a hamiltonian cycle $H^{\prime}$ for $G^{\prime}$. Deleting the vertices of $V^{\prime}-V$ yields a subhamiltonian cycle $H$ for $G$ that crosses each face at most once.

Given an embedded triconnected 4-planar graph $G$ with a fixed outerface and a separating triangle $\mathcal{T}$ with vertices $V(\mathcal{T})=\{A, B, \Gamma\}$, we denote the subgraph of $G$ contained in $\mathcal{T}$ by $G_{\text {in }}(\mathcal{T})$ and the subgraph of $G$ outside $\mathcal{T}$ by $G_{\text {out }}(\mathcal{T})$. We also denote $\bar{G}_{\text {in }}(\mathcal{T})=G-G_{\text {out }}(\mathcal{T})$ and $\bar{G}_{\text {out }}(\mathcal{T})=G-G_{\text {in }}(\mathcal{T})$. Since $G$ is triconnected and 4-planar, every vertex of $\mathcal{T}$ has degree four and is adjacent to exactly one vertex in $G_{\text {in }}(\mathcal{T})$ and $G_{\text {out }}(\mathcal{T})$, respectively. We denote these with $A_{\text {in }}, B_{\text {in }}, \Gamma_{\text {in }}$ and $A_{\text {out }}, B_{\text {out }}, \Gamma_{\text {out }}$, respectively (see Fig. 1 ).

- Lemma 2. Given a 4-planar triconnected graph $G$ and a separating triangle $\mathcal{T}=\{A, B, \Gamma\}$, then $A_{\text {in }}, B_{\text {in }}, \Gamma_{\text {in }}\left(A_{\text {out }}, B_{\text {out }}, \Gamma_{\text {out }}\right)$ are pairwise distinct or all represent the same vertex.

Proof. In the other case, where w.l.o.g. $A_{i n}=B_{i n}=v$ and $\Gamma_{i n} \neq v$, there exists a separation pair $(v, \Gamma)$ contradicting the triconnectivity of $G$. A symmetric argument applies to $A_{\text {out }}, B_{\text {out }}, \Gamma_{\text {out }}$.

- Lemma 3. In a 4-planar triconnected graph, every pair of distinct separating triangles $\mathcal{T}$ and $\mathcal{T}^{\prime}$ is vertex disjoint, i.e. $V(\mathcal{T}) \cap V\left(\mathcal{T}^{\prime}\right)=\emptyset$.

Proof. Assume to the contrary that $\mathcal{T}$ and $\mathcal{T}^{\prime}$ share an edge or a vertex. In the first case, let w.l.o.g. $e=(u, v)$ be the common edge. The degree of both $u$ and $v$ is at least five, since three edges are required for $\mathcal{T}, \mathcal{T}^{\prime}$ and two additional edges to connect $G_{\text {in }}(\mathcal{T})$ and $G_{\text {in }}\left(\mathcal{T}^{\prime}\right)$ to $\mathcal{T}$ and $\mathcal{T}^{\prime}$, respectively. In the second case, let $v$ denote the common vertex. Since $v$ is part of two edge disjoint cycles and connected to $G_{i n}(\mathcal{T})$ and $G_{i n}\left(\mathcal{T}^{\prime}\right)$, it follows that $\operatorname{deg}(v) \geq 6$.

Consider now a 4-planar triconnected graph with a single separating triangle $\mathcal{T}$. Similar to Chen [3], the idea is to compute two cycles $H_{\text {in }}(\mathcal{T})$ and $H_{\text {out }}(\mathcal{T})$ for $\bar{G}_{\text {in }}(\mathcal{T})$ and $\bar{G}_{\text {out }}(\mathcal{T})$ and link them via the separating triangle together. The crucial observation is that if two cycles intersect as illustrated in Fig. 2, i.e., they contain two edges of the triangle but have only one of them in common, then we can always merge them into one cycle.

- Lemma 4. Let $G$ be a triconnected 4-planar graph, $\mathcal{T}$ a separating triangle, and $H_{\text {in }}(\mathcal{T})$ and $H_{\text {out }}(\mathcal{T})$ two subhamiltonian cycles for $\bar{G}_{\text {in }}(\mathcal{T})$ and $\bar{G}_{\text {out }}(\mathcal{T})$, resp. If $E\left(H_{\text {in }}(\mathcal{T})\right) \cap E(\mathcal{T})=$ $\left\{e_{\text {in }}, e\right\}$ and $E\left(H_{\text {out }}(\mathcal{T})\right) \cap E(\mathcal{T})=\left\{e_{\text {out }}, e\right\}$ where $\left\{e, e_{\text {in }}, e_{\text {out }}\right\}$ are the edges of $\mathcal{T}$, then $G$ is subhamiltonian.

Proof. Let w.l.o.g. $e=(A, B), e_{i n}=(B, \Gamma)$ and $e_{\text {out }}=(A, \Gamma)$ as illustrated in Fig. 2. The result of removing the edges of $\mathcal{T}$ from both cycles are two paths $P_{\text {out }}=B \rightsquigarrow \Gamma$ and $P_{\text {in }}=\Gamma \rightsquigarrow A$. Joining them at $\Gamma$ and inserting $e$ yields a subhamiltonian cycle.

It remains to show that we can always find two cycles that satisfy the requirements of Lemma 4. We neglect the degenerated case of Lemma 2 , where $G_{\text {out }}(\mathcal{T})$ or $G_{\text {in }}(\mathcal{T})$ is a single vertex, because finding a cycle in that case is trivial. Consider for example $\bar{G}_{\text {out }}(\mathcal{T})$. To obtain $H_{\text {out }}(\mathcal{T})$, we temporarily replace $\mathcal{T}$ in $\bar{G}_{\text {out }}(\mathcal{T})$ with a single vertex $v_{\mathcal{T}}$ as depicted in Fig. 3a. The resulting graph $G_{\text {out }}^{*}(\mathcal{T})$ remains 4 -planar and triconnected, because $\operatorname{deg}\left(v_{\mathcal{T}}\right)=3$ by construction and any path via $\mathcal{T}$ can use $v_{\mathcal{T}}$ instead. One may argue that this operation may introduce additional separating triangles. However, such a triangle must contain $v_{\mathcal{T}}$ and, therefore, $\operatorname{deg}\left(v_{\mathcal{T}}\right)=4$, a contradiction. Now let us assume that $H_{\text {out }}^{*}(\mathcal{T})$ is a subhamiltonian cycle for $G_{\text {out }}^{*}(\mathcal{T})$. The idea is to reinsert $\mathcal{T}$ and reroute $H_{\text {out }}^{*}(\mathcal{T})$ through $\mathcal{T}$ such that the resulting cycle $H_{\text {out }}(\mathcal{T})$ contains two edges $e_{1}, e_{2} \in E(\mathcal{T})$. 


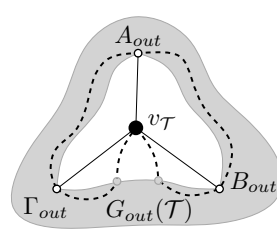

(a) $v_{\mathcal{T}}$ in $G_{\text {out }}^{*}(\mathcal{T})$

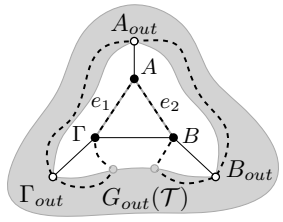

(b) $\mathcal{T}$ in $\bar{G}_{\text {out }}(\mathcal{T})$

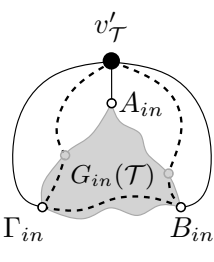

(c) $v_{\mathcal{T}}^{\prime}$ in $G_{i n}^{*}(\mathcal{T}$

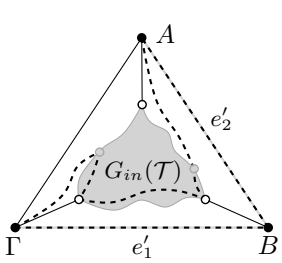

(d) $\mathcal{T}$ in $\bar{G}_{i n}(\mathcal{T})$

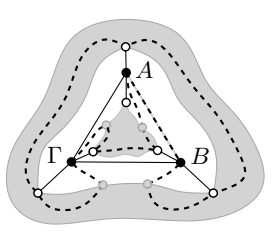

(e) $G$ with $\mathcal{T}$ and $H$

Figure 3 (a) Subhamiltonian cycle $H_{\text {out }}^{*}(\mathcal{T})$ in $G_{\text {out }}^{*}(\mathcal{T})$ containing $v_{\mathcal{T}}$. (b) Augmenting $H_{\text {out }}^{*}(\mathcal{T})$ yields $H_{\text {out }}(\mathcal{T})$ containing edges $e_{1}=(\Gamma, A)$ and $e_{2}=(A, B)$. (c) Dummy vertex $v_{\mathcal{T}}^{\prime}$ as replacement for $\mathcal{T}$ in $G_{i n}^{*}(\mathcal{T})$ and a cycle $H_{\text {in }}^{*}(\mathcal{T})$. (d) Rerouting $H_{\text {in }}^{*}(\mathcal{T})$ through $\mathcal{T}$ resulting in $H_{\text {in }}(\mathcal{T})$ with edges $e_{1}^{\prime}=(\Gamma, B)$ and $e_{2}=(A, B)$. (d) The result of merging $H_{\text {in }}(\mathcal{T})$ and $H_{\text {out }}(\mathcal{T})$ into a cycle $H$ for $G$.

- Lemma 5. Let $G$ be a triconnected 4-planar graph, $\mathcal{T}$ a separating triangle. Furthermore, let $G_{\text {out }}^{*}(\mathcal{T})$ denote the graph resulting from replacing $\mathcal{T}$ by a vertex $v_{\mathcal{T}}$ in $\bar{G}_{\text {out }}(\mathcal{T}) . \quad A$ subhamiltonian cycle $H_{\text {out }}^{*}(\mathcal{T})$ for $G_{\text {out }}^{*}(\mathcal{T})$ can be augmented to a subhamiltonian cycle $H_{\text {out }}(\mathcal{T})$ for $\bar{G}_{\text {out }}(\mathcal{T})$ such that it contains two edges of $\mathcal{T}$, i.e., $E\left(H_{\text {out }}(\mathcal{T})\right) \cap E(\mathcal{T})=\left\{e_{1}, e_{2}\right\}$. If $H_{\text {out }}^{*}(\mathcal{T})$ crosses every face of $G_{\text {out }}^{*}(\mathcal{T})$ at most once, one may choose any pair $e_{1}, e_{2} \in E(\mathcal{T})$ to lie on $H_{\text {out }}(\mathcal{T})$.

Proof. We only sketch the proof. It is sufficient to consider every combination of $e_{1}, e_{2}$ and the location of the predecessor and successor of $v_{\mathcal{T}}$ in $H_{\text {out }}^{*}(\mathcal{T})$. It immediately becomes clear that in almost every situation $H_{\text {out }}^{*}(\mathcal{T})$ can be rerouted through $\mathcal{T}$ such that the resulting cycle $H_{\text {out }}(\mathcal{T})$ contains two prescribed edges $e_{1}, e_{2}$ of $\mathcal{T}$. Only in one case, where $H_{\text {out }}^{*}(\mathcal{T})$ crosses an incident face twice to visit $v_{\mathcal{T}}$, a specific combination of edges is required.

In the single-separating triangle scenario, both $\bar{G}_{\text {out }}(\mathcal{T})$ and $\bar{G}_{\text {in }}(\mathcal{T})$ are free of separating triangles. Therefore, we may construct two graphs $G_{\text {out }}^{*}(\mathcal{T}), G_{\text {in }}^{*}(\mathcal{T})$ by replacing $\mathcal{T}$ with dummy vertices. Applying Lemma 1 to them yields two subhamiltonian cycles $H_{\text {out }}^{*}(\mathcal{T})$ and $H_{\text {in }}^{*}(\mathcal{T})$, both crossing every face of $G_{\text {out }}^{*}(\mathcal{T})$ and $G_{\text {in }}^{*}(\mathcal{T})$ at most once. Hence, we may augment them with the aid of Lemma 5 such that they contain each two edges of $\mathcal{T}$. By choosing the combination of edges such that $H_{\text {out }}(\mathcal{T})$ and $H_{\text {in }}(\mathcal{T})$ meet the requirements of Lemma 4, we can merge them into a single subhamiltonian cycle $H$ for $G$.

While the property that $G_{\text {out }}^{*}(\mathcal{T})$ and $G_{\text {in }}^{*}(\mathcal{T})$ are both free of separating triangles enables us to conveniently choose two edges for each cycle $H_{\text {out }}(\mathcal{T}), H_{\text {in }}(\mathcal{T})$, this only works for a single separating triangle. However, a closer look reveals that it is sufficient to have a choice for either $H_{\text {out }}(\mathcal{T})$ or $H_{\text {in }}(\mathcal{T})$, not necessarily both of them. The idea is to first augment the cycle for which we do not have a choice to see which edges of $\mathcal{T}$ are part of it, then we choose the edges for the second cycle accordingly. We summarize the idea as the main result of this section and describe it in a more formal manner in form of a proof.

- Theorem 6. Every triconnected 4-planar graph is subhamiltonian.

Proof. Let $G$ denote a triconnected 4-planar graph and $\tau(G)$ the number of separating triangles in $G$. We prove by induction and claim that for any $\tau(G) \geq 0$, we can compute a subhamiltonian cycle $H$ for $G$. Base case: Since $\tau(G)=0$, we can directly apply Lemma 1 . Inductive case: For $\tau(G)>0$, we pick a separating triangle $\mathcal{T}$ such that $\tau\left(\bar{G}_{i n}(\mathcal{T})\right)=0$. Let $G_{\text {out }}^{*}(\mathcal{T})$ be the result of replacing $\mathcal{T}$ by $v_{\mathcal{T}}$ in $\bar{G}_{\text {out }}(\mathcal{T})$. Notice that $\tau\left(G_{\text {out }}^{*}(\mathcal{T})\right)=\tau(G)-1$ holds. Hence, by induction hypothesis, $G_{\text {out }}^{*}(\mathcal{T})$ has a subhamiltonian cycle $H_{\text {out }}^{*}(\mathcal{T})$. We 
reinsert $\mathcal{T}$ and augment $H_{\text {out }}^{*}(\mathcal{T})$ such that the result $H_{\text {out }}(\mathcal{T})$ contains two (arbitrary) edges $e_{1}, e_{2}$ of $\mathcal{T}$. In a similar way, we replace $\mathcal{T}$ in $\bar{G}_{i n}(\mathcal{T})$ by $v_{\mathcal{T}}^{\prime}$ to obtain $G_{i n}^{*}(\mathcal{T})$. Since $\tau\left(\bar{G}_{i n}(\mathcal{T})\right)=\tau\left(G_{i n}^{*}(\mathcal{T})\right)=0$ holds, we can apply Lemma 1 to $G_{i n}^{*}(\mathcal{T})$ and compute a cycle $H_{i n}^{*}(\mathcal{T})$ that crosses each face at most once. With Lemma 5 we may obtain a cycle $H_{\text {in }}(\mathcal{T})$ for $\bar{G}_{i n}(\mathcal{T})$ with two edges $e_{1}^{\prime}, e_{2}^{\prime} \in E(\mathcal{T})$ of our choice. Choosing $e_{1}^{\prime}=e_{1}$ and $e_{2}^{\prime} \neq e_{2}$ yields two cycles $H_{\text {out }}(\mathcal{T}), H_{\text {in }}(\mathcal{T})$ that meet the requirements of Lemma 4 and we can merge them into one cycle $H$ for $G$.

The proof of Theorem 6 is constructive. Embedding $G$ and identifying all separating triangles in $G$ can be done in linear time. Augmenting a cycle and merging two of them takes constant time. Disjointness of separating triangles yields a linear number of subproblems and every edge occurs in at most one such subproblem. Hence, the total time spent for the subroutine of Lemma 1 is linear in the size of $G$.

- Corollary 7. A subhamiltonian cycle of a triconnected 4-planar graph can be found in linear time.

\section{Two-Page Book Embeddings of General 4-Planar Graphs}

In this section, we prove that any planar graph of maximum degree 4 admits a two-page book embedding. W.l.o.g. we assume that the input graph $G$ is biconnected, since it is known that the page number of a graph equals the maximum of the page number of its biconnected components [2]. One can also neglect the exact geometry, as two edges that are drawn on the same page cross iff their endpoints alternate along the spine. We say that an edge $e$ nests a vertex $v$ iff one endpoint of $e$ is to the left of $v$ along the spine and the other endpoint of $e$ to its right. We also say that an edge $e$ nests an edge $e^{\prime}$ iff both $e$ and $e^{\prime}$ are drawn on the same page and both endpoints of $e^{\prime}$ are nested by $e$. Observe that nested edges do not cross.

Our approach is as follows: First remove from $G$ cycle $C_{\text {out }}$ delimiting the outerface of $G$ and contract each bridge-block of the remaining graph into a single vertex. Let $F$ be the implied graph, which is a forest, as $G-C_{\text {out }}$ is not necessarily connected. $C_{\text {out }}$ is embedded, s.t.: (i) the order of the vertices of $C_{\text {out }}$ along the spine is fixed (and follows the one in which the vertices of $C_{\text {out }}$ appear along $C_{\text {out }}$ ), and, (ii) all edges of $C_{\text {out }}$ are on the same page, except for the one that connects its outermost vertices. Then we describe how to embed without crossings: (i) the chords of $C_{\text {out }}$, (ii) forest $F$, and, (iii) the edges between $C_{\text {out }}$ and $F$. To obtain a two-page book embedding of $G$, we replace each vertex of $F$ with a cycle (embedded similarly to $C_{\text {out }}$ ), whose length equals to the length of the cycle delimiting the outerface of the bridge-block it corresponds to in $G-C_{\text {out }}$, and recursively embed its interior.

More formally, consider an arbitrary simple cycle $C: v_{1} \rightarrow v_{2} \rightarrow \ldots \rightarrow v_{k} \rightarrow v_{1}$ of $G$. The removal of $C$ results in two planar subgraphs $G_{i n}(C)$ and $G_{\text {out }}(C)$ of $G$ that are the components of $G-C$ that lie in the interior and exterior of $C$ in $G$, resp. Note that $G_{i n}(C)$ and $G_{\text {out }}(C)$ are not necessarily connected. Let $\bar{G}_{i n}(C)\left(\bar{G}_{\text {out }}(C)\right.$, resp. $)$ be the subgraph of $G$ induced by $C$ and $G_{i n}(C)\left(G_{\text {out }}(C)\right.$, resp.). For the recursive step, we assume the following invariant properties:

IP-1: The order of the vertices of $\bar{G}_{\text {out }}(C)$ along the spine $\ell$ is fixed and the page in which each edge of $\bar{G}_{\text {out }}(C)$ is drawn (i.e., top- or bottom-drawn) is determined s.t. the book embedding of $\bar{G}_{\text {out }}(C)$ is planar. In other words, we assume that we have already produced a two-page book embedding for $\bar{G}_{\text {out }}(C)$, in which no edge crosses the spine.

IP-2: The combinatorial embedding of $\bar{G}_{\text {out }}(C)$ is consistent with a given planar combinatorial embedding of $G$. 
IP-3: The vertices of $C$ occupy consecutive positions along $\ell$, s.t. $v_{1}\left(v_{k}\right.$, resp.) is the leftmost (rightmost, resp.) along $\ell$. Moreover, all edges of $C$ are on the same page, except for the one that connects $v_{1}$ and $v_{k}$. Say w.l.o.g. that $\left(v_{1}, v_{k}\right)$ is on the top-page (or top-drawn), while the remaining edges of $C$, namely edges $\left(v_{i}, v_{i+1}\right)$ for $1 \leq i<k$, are on the bottom-page (or bottom-drawn).

IP-4: If $C$ is not identified with the cycle delimiting the outerface of $G$, the degree of either $v_{1}$ or $v_{k}$ is at most 3 in $\bar{G}_{i n}(C)$. Say w.l.o.g. that $v_{k}$ is of degree at most 3.

IP-5: If vertex $v_{1}$ has degree 4 in $\bar{G}_{i n}(C)$, then it is adjacent to zero or two chords of $C$.

We note that the combinatorial embedding specified in IP-2 is maintained throughout the whole drawing process. This combined with the fact that every edge entirely lies on one page is sufficient to ensure planarity. Note that we first present the recursive step of our algorithm and then its base, as this approach shows better how the different ideas flow one after the other. Let $v_{i}$ be a vertex of $C, i=1, \ldots, k$. Since $G$ is of max-degree $4, v_{i}$ is incident to at most two undrawn edges. Assume that $v_{i}$ has at least one undrawn edge. We refer to the edge incident to $v_{i}$ that follows $\left(v_{i}, v_{(i+1)} \bmod k\right)$ in the counterclockwise order of the edges around $v_{i}$ (as defined by the combinatorial embedding specified by IP-2), as the right edge of $v_{i}$. If $v_{i}$ is adjacent to two undrawn edges, then the one that is not identified with the right edge of $v_{i}$ is its left edge; otherwise, the left and the right edge of $v_{i}$ are identified.

Initially, we draw the chords of $C$ on the top-page. By IP-2 and IP-3, no two chords intersect. We then draw $G_{i n}(C)$ and the edges between $C$ and $G_{i n}(C)$. Note that $G_{i n}(C)$ is not necessarily connected. Hence, its bridge-block trees form a forest. As already stated, we contract each bridge-block of $G_{i n}(C)$ into a single vertex, which we call block-vertex; see Figs. 4a-4b. We distinguish two types of block-vertices: those adjacent to vertices of $C$ (anchors) and those adjacent to other block-vertices only (ancillaries). From the contraction, it follows that an edge between $C$ and a certain anchor can be of multiplicity at most two. Edges among block-vertices are always simple. We will first determine the positions of all anchors along $\ell$. Consider an anchor $c$, then among the edges between $c$ and $C$, we select and mark exactly one, s.t.: (i) the marked edge will be drawn on the bottom-page and (ii) all other edges incident to $c$ (i.e., either edges between $c$ and $C$ that are not marked, or between $c$ and block-vertices) will be drawn on the top-page. Let $v_{l, c}$ be the leftmost vertex of $C$ adjacent to $c$ along $\ell$. If $\left(c, v_{l, c}\right)$ is simple, we select and mark this edge. Otherwise, we mark the right edge of $v_{l, c}$. Hence, each anchor has exactly one marked edge and each vertex of $C$ is incident to at most two marked edges. Let $v \in C$ be a vertex of $C$ adjacent to at least one anchor through a marked edge. We distinguish two cases:

Case $1 v$ is adjacent to exactly two anchors $c$ and $c^{\prime}$ through two marked edges $e$ and $e^{\prime}$, resp.: Assume w.l.o.g. that $e$ is the left edge of $v$ and $e^{\prime}$ its right edge. Then, both $c$ and $c^{\prime}$ are placed directly to the right of $v$ and $c$ precedes $c^{\prime}$ (see Fig. 4d). Note that $v$ cannot be the rightmost vertex of $C$ due to IP-4.

Case $2 v$ is adjacent to one anchor $c$ through a marked edge e: If $\operatorname{deg}(v)=3$ in $\bar{G}_{i n}(C)$, then we distinguish two sub-cases. If $v$ is not the rightmost vertex of $C$, then $c$ is placed directly to the right of $v$ (see Fig. 4e). Otherwise, directly to its left (see Fig. 4f). It now remains to consider the case where $\operatorname{deg}(v)=4$ in $\bar{G}_{i n}(C)$. In this case, by IP-4 it follows that $v$ is not the rightmost vertex of $C$. Again, we distinguish two sub-cases:

- If e is the right edge of $v$, then $c$ is placed directly to the right of $v$ (see Fig. $4 \mathrm{~g}$ ).

- If $e$ is the left edge of $v$, then $c$ is placed directly to the left of $v$ (see Fig. $4 \mathrm{~h}$ ); $v$ cannot be the leftmost vertex of $C$, as the right edge of $v$ would be a chord, violating IP-5. 


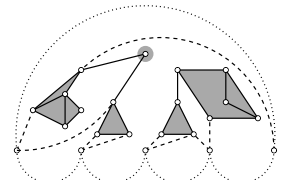

(a) Bridge-blocks of $G_{\text {in }}(C)$

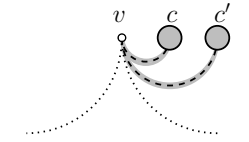

(d)

(e)

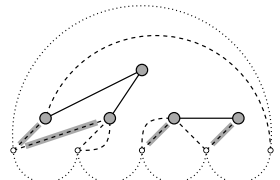

(b) Forest of blockvertices

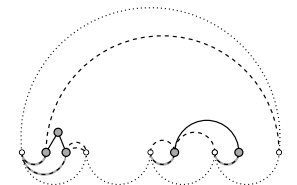

(c) Placement of anchors
Figure 4 In all figures, the edges of $C$ are drawn dotted, bridge-blocks are colored gray and edges between $C$ and anchors are drawn dashed; marked edges are highlighted in gray.

All marked edges are bottom-drawn. Edges between anchors and $C$ that are not marked are top-drawn; see Fig. 4c. Observe that we do not change the underlying combinatorial embedding of $G$, preserving IP-2. Hence, the book embedding constructed so far is planar.

Now observe that ancillaries form a new forest (forest of ancillaries), which is a subgraph of the initial forest containing all block-vertices. Let $T$ be a tree of the forest of ancillaries and let $c_{1}, \ldots, c_{t}$ be anchors that (i) are adjacent to at least one ancillary of $T$, and (ii) $c_{i}$ is to the left of $c_{i+1}, i=1, \ldots, t-1$. We refer to $c_{1}, \ldots, c_{t}$ as the anchors of $T$, and to the tree formed by $T$ and its anchors as the anchored tree of $T$, denoted by $\bar{T}$. We say that two anchors of $\bar{T}$ are consecutive iff there is no anchor of $\bar{T}$ between them.

- Lemma 8. For anchored trees the following hold: $(i)$ Two trees $\bar{T}$ and $\overline{T^{\prime}}$ share at most a common anchor; (ii) $\bar{T}$ contains at least two anchors; and (iii) every leaf of $\bar{T}$ is an anchor of $\bar{T}$, and vice versa.

Proof. The assumption that one of the two properties does not hold contradicts either the connectivity or biconnectivity of $G$.

Assume now that $\bar{T}$ is rooted at anchor $c_{1}$ (rooted anchored tree). For an anchor or ancillary $c$ of $\bar{T}$, denote by $p(c)$ the parent of $c$ in $\bar{T}$ and let $p\left(c_{1}\right)$ be any of the vertices of $C$ adjacent to $c_{1}$. For an ancillary $c$ of $T$ (i.e., non-leaf in $\bar{T}$ ), we define an order for its children: if $c^{\prime}$ and $c^{\prime \prime}$ are children of $c$, then $c^{\prime}<c^{\prime \prime}$ iff $c^{\prime}$ precedes $c^{\prime \prime}$ in the counterclockwise order of the edges around $c$ (defined by the combinatorial embedding specified by IP-2), when starting from $(c, p(c))$. By this order, we label the vertices of $\bar{T}$ as they appear in the pre-order traversal of $\bar{T}$ (labeled anchored tree); see Fig.5a.

- Lemma 9. For each ancillary c of a labeled anchored tree $\bar{T}$ there is (i) at least an anchor of $\bar{T}$ with label smaller than that of $c$ and (ii) at least another with label greater than that of $c$

Proof. The leftmost anchor (i.e. root) of $\bar{T}$ is zero labeled, which proves (i). The greatest labeled vertex of $\bar{T}$ is a leaf of $\bar{T}$ (due to pre-order traversal) and by Lemma 8(iii) an anchor of $\bar{T}$ which proves (ii).

We first define the order in which the trees of the forest of ancillaries will be drawn. Let $G_{a u x}^{T}$ be an auxiliary graph whose vertices correspond to trees and there is a directed edge $\left(v_{T^{\prime}}, v_{T}\right)$ in $G_{a u x}^{T}$ iff $\overline{T^{\prime}}$ has an anchor between two consecutive anchors of $\bar{T}$. The desired order is defined by a topological sorting of $G_{a u x}^{T}$, which exists due to the following lemma. 


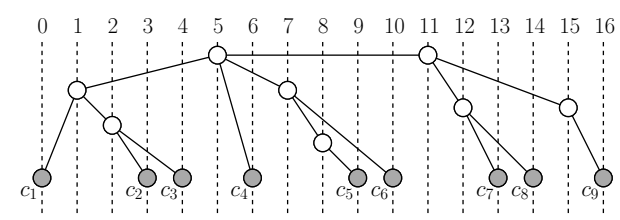

(a) A labeled anchored tree $\bar{T}$

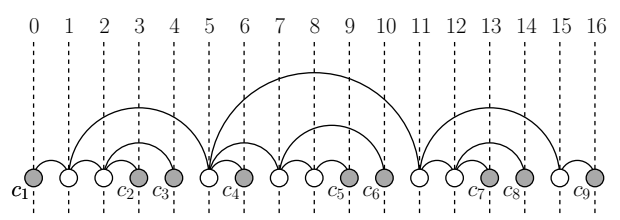

(b) The placement of the ancillaries of $\bar{T}$

Figure 5 In both figures, anchors are colored gray; the indices of the vertical grid-lines denote the labeling of $\bar{T}$.

- Lemma 10. Auxiliary graph $G_{a u x}^{T}$ is a directed acyclic graph.

Proof. Assume to the contrary that there is a cycle $v_{T_{1}} \rightarrow \ldots v_{T_{s}} \rightarrow v_{T_{1}}$ in $G_{a u x}^{T}$. Let $I_{i}$ be the interval defined by the left/right-most anchors of $\overline{T_{i}}$. Edge $\left(v_{T_{i}}, v_{T_{i+1}}\right)$ implies that there is an anchor of $\overline{T_{i}}$ between consecutive anchors of $\overline{T_{i+1}}$. However, in this case all anchors of $\overline{T_{i}}$ should be between the same two anchors of $\overline{T_{i+1}}$, as otherwise the embedding specified by IP-2 is not planar. So, $I_{i} \subseteq I_{i+1}$. By Lemma 8(i), it follows that $I_{i} \neq I_{i+1}$. Hence, $I_{1} \subset \ldots \subset I_{s} \subset I_{1}$, a contradiction.

Lemma 10 implies that drawing the trees in the order defined by a topological sorting of $G_{a u x}^{T}$, assures that the tree $T^{\prime}$ will be drawn before $T$, if $\overline{T^{\prime}}$ has an anchor that is between two consecutive anchors of $\bar{T}$ along $\ell$. Now assume that we have drawn zero or more of these trees s.t. (i) all edges are top-drawn, (ii) there are no edge crossings, and (iii) the combinatorial embedding specified by IP-2 is preserved. Let $T$ be the next tree to be drawn. The following lemma presents an important property of our drawing approach.

- Lemma 11. Assume that all trees that precede $T$ in a topological sorting of $G_{\text {aux }}^{T}$ have been drawn on the top-page without edge crossings by preserving the combinatorial embedding specified by IP-2. If $e$ is a top-drawn edge that does not belong to $\bar{T}$ and nests at least one anchor of $\bar{T}$, then it nests all anchors of $\bar{T}$.

Proof. The detailed proof is based on a case-analysis on the type of edge $e$ : $(i)$ top-drawn edge of $C$; (ii) edge of an anchored tree $\overline{T^{\prime}}$ drawn before $\bar{T}$; and (iii) not an edge of a previously drawn anchored tree (i.e., each endpoint of $e$ is either a vertex of $C$ or an anchor).

We now describe how to draw $T$ on the top page s.t. (i) there are no edge crossings, and, (ii) the combinatorial embedding specified by IP-2 is preserved. More precisely, we place each ancillary $c$ of $T$ between a pair of consecutive anchors of $\bar{T}$, s.t. the label of $c$ is larger (smaller) than the label of the anchor to its left (right) ${ }^{1}$; for ancillaries placed between the same pair of anchors, the one with smaller label is to the left; all edges of $\bar{T}$ are top-drawn (see Fig.5b). Note that we have not fully specified the exact positions of the ancillaries of $\bar{T}$ along $\ell$, since between consecutive anchors of $\bar{T}$ there may exist anchors that do not belong to $\bar{T}$ or vertices of $C$ or anchors/ancillaries of trees that have already been drawn. Details will be given shortly. Notice that all ancillaries of $\bar{T}$ are placed between its left/right-most anchors, which by Lemma 11 implies that if a top-drawn edge (that does not belong to $\bar{T}$ ) nests at least one anchor of $\bar{T}$, then it nests the entire tree $\bar{T}$. By exploiting the correspondence between the left-to-right order of the vertices of $\bar{T}$ along $\ell$ and the labeling of $\bar{T}$, we can prove that the drawing of $\bar{T}$ is planar.

\footnotetext{
1 Note that the existence of this pair of consecutive anchors of $\bar{T}$ is implied by Lemma 9 .
} 
- Lemma 12. The drawing of the anchored tree $\bar{T}$ is planar.

Proof. Assume to the contrary that $e=\left(c_{1}, c_{2}\right)$ and $e^{\prime}=\left(c_{1}^{\prime}, c_{2}^{\prime}\right)$ of $\bar{T}$ cross. Since $e$ and $e^{\prime}$ are top-drawn, their endpoints alternate along $\ell$. Let the order on $\ell$ be $c_{1} \rightarrow c_{1}^{\prime} \rightarrow c_{2} \rightarrow c_{2}^{\prime}$. Hence, $c_{1}$ is the parent of $c_{2}$, as the label of $c_{1}$ is smaller than that of $c_{2}$ and they are adjacent in $\bar{T}$. Similarly, $c_{1}^{\prime}$ is the parent of $c_{2}^{\prime}$. Since between $c_{1}$ and $c_{2}$ are drawn subtrees of $\bar{T}$ rooted at children of $c_{1}$ other than $c_{2}, c_{1}^{\prime}$ and $c_{2}^{\prime}$ belong to a subtree rooted at a child of $c_{1}$, different from $c_{2}$, which implies that the label of $c_{2}^{\prime}$ is smaller than that of $c_{2}$, a contradiction.

Recall that we have not fully specified the exact positions of the ancillaries of $\bar{T}$ along $\ell$. Consider the following scenario. There is a path $P$ of top-drawn edges (e.g., non-marked edges incident to $C$ and/or edges of previously drawn trees) joining a pair of consecutive anchors of $\bar{T}$ and our algorithm must place an ancillary $c$ of $\bar{T}$ between them. Since $c$ is nested by an edge of $P$ and all edges of $\bar{T}$ are top-drawn, an edge connecting $c$ with an ancillary of $\bar{T}$ placed between another pair of consecutive anchors of $\bar{T}$ will cross $P$. The following lemma ensures that this scenario cannot occur, as such a path cannot exist.

- Lemma 13. Let $u_{0}, u_{1}, \ldots, u_{l+1}, l \geq 0$, be vertices (anchors/ancillaries are treated as vertices) drawn on $\ell$ from left to right, s.t. $u_{0}$ and $u_{l+1}$ are two consecutive anchors of $\bar{T}$. Assume that all trees anchored at $u_{1}, \ldots, u_{l}$ have been drawn on the top-page without edge crossings by preserving the combinatorial embedding specified by IP-2, while T has not been drawn. Then, there is an index $i \in\{0,1, \ldots, l\}$, such that no two adjacent vertices $u_{k}$ and $u_{m}$ exist with $0 \leq k \leq i, i+1 \leq m \leq l+1$ and $\left(u_{k}, u_{m}\right)$ is top-drawn.

Proof. Assume to the contrary that for all $i \in\{0, \ldots, l\}$, there are two adjacent vertices $u_{k}$ and $u_{m}$ with $0 \leq k \leq i, i+1 \leq m \leq l+1$ and $\left(u_{k}, u_{m}\right)$ is on the top-page. One can prove that there is a top-drawn path $P\left(u_{0} \rightarrow u_{l+1}\right): u_{0} \rightarrow u_{j_{1}} \ldots u_{j_{p}} \rightarrow u_{l+1}$ consisting of vertices of $\left\{u_{0}, \ldots, u_{l+1}\right\}$, whose edges are top-drawn and for each edge of $P\left(u_{0} \rightarrow u_{l+1}\right)$ there is not a top-drawn edge with endpoints in $\left\{u_{0}, \ldots, u_{l+1}\right\}$ that nests it. However, the existence of $P\left(u_{0} \rightarrow u_{l+1}\right)$ implies that $G$ should contain a vertex of degree five, a contradiction.

We are now ready to specify the exact positions of the ancillaries of $\bar{T}$ along $\ell$. Assume that a particular number of ancillaries of $\bar{T}$ should be drawn between two consecutive anchors $c_{i}$ and $c_{i+1}$ of $\bar{T}, i=1, \ldots, t-1$. By Lemma 13, there is a pair of vertices that are between $c_{i}$ and $c_{i+1}$ along $\ell$ and there is not a top-drawn edge with endpoints between $c_{i}$ and $c_{i+1}$ nesting both of these vertices. We place between this pair of vertices all ancillaries of $\bar{T}$ that must reside between $c_{i}$ and $c_{i+1}$, without changing their relative order, i.e., for ancillaries placed between $c_{i}$ and $c_{i+1}$, the one with smaller label is to the left. Lemma 12 ensures the planarity of $\bar{T}$. It remains to prove that the combinatorial embedding specified by IP-2 is preserved.

- Lemma 14. Assume that all trees that precede $T$ in a topological sorting of $G_{a u x}^{T}$ have been drawn on the top-page without edge crossings by preserving the combinatorial embedding specified by IP-2. When $\bar{T}$ is drawn, the combinatorial embedding specified by IP-2 is also preserved.

Proof. We only sketch the proof. Since the drawing of $\bar{T}$ preserves the order of the edges around all ancillaries, the combinatorial embedding specified by IP-2 is preserved for all ancillaries of $\bar{T}$. Then, one can prove that this property is propagated to all vertices of $T$.

The following lemma focuses on the case where $C$ contains a vertex with degree 2 in $\bar{G}_{i n}(C)$ (other than its leftmost or rightmost vertex). We will utilize this lemma later. 


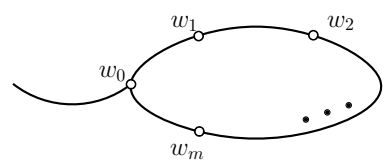

(a)

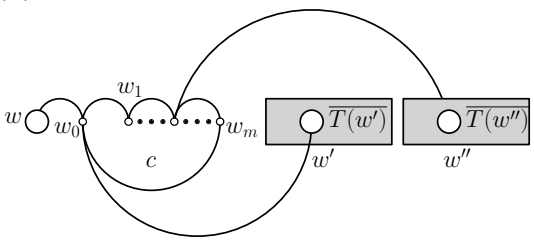

(d)

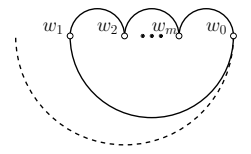

(b)

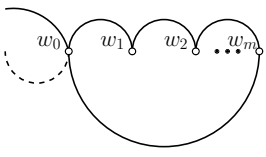

(c)

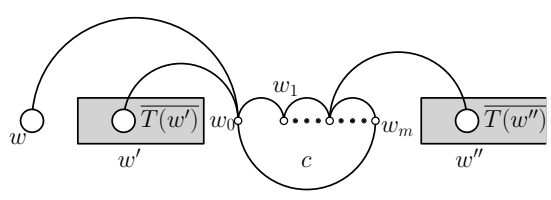

(e)

Figure 6 (a) The outerface of a block-vertex $c$. (b)-(c) different cases that occur when drawing the outerface of $c$, in the case where $c$ is anchor. (d) Ancillary $c$ needs to be repositioned. (e) Its placement is determined by Lemma 16.

- Lemma 15. Let $v$ be a vertex of $C$ with degree 2 in $\bar{G}_{\text {in }}(C)$ that is not the left/right-most vertex of $C$. Let also $v_{r}\left(v_{l}\right)$ be its next neighbor on $C$ to its right (left resp.). Since edge $\left(v, v_{r}\right)$ belongs to $C$, it is drawn on the bottom-page. However, it can also be drawn on the top-page without edge-crossings, while the combinatorial embedding specified by IP-2 is maintained.

Proof. We only sketch the proof. First observe that if no block-vertex is drawn between $v$ and $v_{r}$, then obviously $\left(v, v_{r}\right)$ can be drawn on the top-page. Otherwise, one can move the block-vertices in between to the left of $v$, so that $v$ and $v_{r}$ are consecutive along $\ell$.

Up to now, we have drawn $\bar{G}_{i n}(C)$, s.t., every bridge-block of $G_{i n}(C)$ is contracted to a block-vertex that lies on $\ell$ and each edge is drawn either on the bottom (if it is a marked edge) or on the top-page (otherwise). Next, we describe how to recursively proceed. Let $c$ be a block-vertex of $G_{i n}(C)$ with outerface $\mathcal{F}_{c}$. Initially, assume that $\mathcal{F}_{c}$ is a simple cycle. If $c$ is an anchor, denote by $w_{0}$ the vertex of $\mathcal{F}_{c}$ incident to the marked edge of $c$. If $c$ is an ancillary, then $c$ belongs to an anchored tree. In this case, $w_{0}$ denotes the vertex of $\mathcal{F}_{c}$ adjacent to the closest neighbor of $c$ to its left, which is well-defined since $c$ is always placed between two consecutive anchors of the anchored tree it belongs to. Let $w_{0}, w_{1}, \ldots, w_{m}$ be the vertices of $\mathcal{F}_{c}$, in the clockwise traversal of $\mathcal{F}_{c}$ from $w_{0}$ (see Fig. 6a).

If $c$ is an anchor (i.e., $w_{0}$ is incident to a marked edge), then we place the vertices of $\mathcal{F}_{c}$ on $\ell$ as follows: (i) $w_{0}$ occupies the position of $c$ and it is the rightmost vertex of $\mathcal{F}_{c}$ on $\ell$, (ii) $w_{1}$ is the leftmost vertex of $\mathcal{F}_{c}$ on $\ell$, (iii) $w_{i}$ is to the left of $w_{i+1}$ for $i=1, \ldots, m-1$, and, (iv) there are no vertices in between; see Fig. 6b. All edges of $\mathcal{F}_{c}$ are top-drawn, except for $\left(w_{1}, w_{0}\right)$. This placement is feasible, except for the case in which in the combinatorial embedding specified by IP-2 there is an edge incident to $w_{0}$ that is between $\left(w_{0}, w_{1}\right)$ and the marked edge incident to $w_{0}$ in the counterclockwise order of the edges around $w_{0}$ when starting from $\left(w_{0}, w_{1}\right)$; see Fig. $6 \mathrm{c}$. In this case, we place $w_{0}$ to the left of $w_{1}, \ldots, w_{m}$, s.t. $w_{0}$ is the leftmost vertex of $\mathcal{F}_{c}$. So, $\left(w_{0}, w_{m}\right)$ is the bottom-drawn edge of $\mathcal{F}_{c}$.

Suppose now that $c$ is an ancillary. Let $w$ be the closest neighbor of $c$ to its left on $\ell$. $w$ is the parent of $c$ in the tree in which $c$ belongs to and $\left(w_{0}, w\right)$ is top-drawn. We place the vertices of $\mathcal{F}_{c}$ as follows: (i) $w_{0}$ occupies the position of $c$ and it is the leftmost vertex of $\mathcal{F}_{c}$ on $\ell$, (ii) $w_{m}$ is the rightmost vertex of $\mathcal{F}_{c}$ on $\ell$, (iii) $w_{i}$ is to the left of $w_{i+1}, i=1, \ldots, m-1$, and, (iv) there are no vertices in between. All edges of $\mathcal{F}_{c}$ are top-drawn, except for $\left(w_{0}, w_{m}\right)$. This placement is infeasible only when in the combinatorial embedding specified by IP-2 there 
is an edge incident to $w_{0}$, say $\left(w_{0}, w^{\prime}\right)$, and between $\left(w_{0}, w_{m}\right)$ and $\left(w_{0}, w\right)$ in the clockwise order of the edges around $w_{0}$ when starting from $\left(w_{0}, w_{m}\right)$; see Fig. 6d. Since $c$ has only its parent to its left among the block-vertices of the anchored tree it belongs to, it follows that, $w^{\prime}$ is to the right of $c$. So, $\left(w_{0}, w^{\prime}\right)$ cannot be drawn on the top-page, without deviating the combinatorial embedding specified by IP-2. Since $G$ is biconnected, $c$ is adjacent to at least another block-vertex, say $w^{\prime \prime}$, s.t. $w^{\prime \prime} \notin\left\{w, w^{\prime}\right\}$. The following lemma takes care of this case.

- Lemma 16. Ancillary c can be repositioned on $\ell$, s.t.: (i) $c$ is placed between two consecutive anchors of $\bar{T}$. (ii) The combinatorial embedding specified by IP-2 is preserved and the edges $\left(w_{0}, w\right),\left(w_{0}, w^{\prime}\right)$ and $\left(c, w^{\prime \prime}\right)$ are top-drawn and crossing-free. (iii) $w_{0}$ is leftmost vertex of $\mathcal{F}_{c}$ and $w_{i}$ is to the left of $w_{i+1}, i=1, \ldots, m-1$; All edges of $\mathcal{F}_{c}$ are top-drawn, except for $\left(w_{0}, w_{m}\right)$.

Proof. $w$ is the parent of $c$ and $w^{\prime}, w^{\prime \prime}$ are children of $c$ in $\bar{T}$, with $w^{\prime}$ being the first child of $c$. For our proof, $w^{\prime \prime}$ is its second child. So, $(c, w),\left(c, w^{\prime}\right)$ and $\left(c, w^{\prime \prime}\right)$ are consecutive around $c$ as in Fig. 6d. Let $\overline{T\left(w^{\prime}\right)}$ and $\overline{T\left(w^{\prime \prime}\right)}$ be subtrees of $\bar{T}$ rooted at $w^{\prime}$ and $w^{\prime \prime}$, resp. $c$ is to the left of all vertices of $\overline{T\left(w^{\prime}\right)}$, all vertices of $\overline{T\left(w^{\prime}\right)}$ are to the left of all vertices of $\overline{T\left(w^{\prime \prime}\right)}$ and there are no ancillaries of $\bar{T}$ in between. We place $c$ between the rightmost (leftmost) anchor of $\overline{T\left(w^{\prime}\right)}\left(\overline{T\left(w^{\prime \prime}\right)}\right)$; see Fig. 6e. So, $c$ is placed between two consecutive anchors of $\bar{T}$. If we place the vertices of $\mathcal{F}_{c}$, with $w_{0}$ being leftmost on $\mathcal{F}_{c}$ and $w_{i}$ to the left of $w_{i+1}$, then $\left(w_{0}, w\right),\left(w_{0}, w^{\prime}\right)$ and $\left(c, w^{\prime \prime}\right)$ are drawn on the top-page and the embedding is preserved.

If we process all ancillaries that have to be repositioned from right to left along $\ell$, then by Lemma 16 we obtain a planar drawing in which the embedding specified by IP-2 is preserved once the outerface of each block-vertex is drawn and all edges that connect block-vertices are eventually drawn on the toppage. Initially, we assumed that $\mathcal{F}_{c}$ is simple. If not so, $\mathcal{F}_{c}$ consists of smaller simple subcycles, s.t. (i) any two subcycles share at most one vertex of $\mathcal{F}_{c}$ and (ii) any vertex of $\mathcal{F}_{c}$ is incident to at most two subcycles. Hence, the "tangency graph" of these subcycles (which has a vertex for each subcycle and an edge between every pair of subcycles that share a vertex)

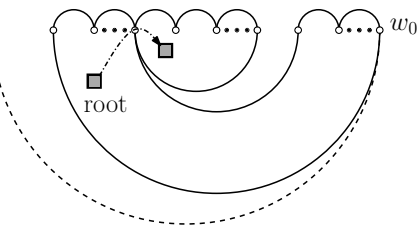

Figure $7 \mathcal{F}_{c}$ is not simple. is a tree. Define $w_{0}$ as in the case of simple cycle and let the tangency tree be rooted at the cycle containing $w_{0}$. Due to degree restriction, $w_{0}$ cannot be incident to two subcycles. We draw the subcycles of $\mathcal{F}_{c}$ in the order implied by the Breadth First Search (BFS) traversal of the tangency tree. The first one (incident to $w_{0}$ ) is drawn as in the case of simple cycle. Each next subcycle is plugged into the drawing, as in Fig. 7.

It remains to ensure that IP-1 up to IP-5 are satisfied when a simple cycle, say $C_{s}$, is recursively drawn. IP-1 holds, since each edge is drawn either on the bottom (if it is a marked edge) or on the top-page (otherwise) and no two edges intersect. Lemma 14 implies IP-2. If $C_{s}$ is the outerface of a block-vertex or a leaf in the tangency tree, then IP-3 trivially holds. If $C_{s}$ is a non-leaf in the tangency tree, it contains at least one edge on the bottom-page (see Fig. 7). This violates IP-3. However, we can benefit from Lemma 15 since the edge which is improperly bottom-drawn is incident to a vertex (of degree four) that is not adjacent to any other vertex in the interior of $C_{s}$. For the sake of the recursion we assume that it is drawn on the top-page and once $C_{s}$ is completely drawn, we redraw it on the bottom-page using Lemma 15. If $C_{s}$ is the outerface of a block-vertex or root of the tangency tree of a non-simple outerface $\mathcal{F}_{c}$, then at least one vertex of $C_{s}$ is adjacent to $G_{\text {out }}\left(C_{s}\right)$. If $C_{s}$ is an internal node of the tangency tree of $\mathcal{F}_{c}$, then its leftmost vertex has two edges in $G_{\text {out }}\left(C_{s}\right)$. Hence, IP-4 also holds. Note that IP-5 does not necessarily hold. However, we can identify a 
maximal separating path of chords of $C_{s}$ adjacent to its leftmost vertex and use it to create two subinstances, which can be recursively drawn; refer to [1] for details.

The recursion begins by specifying a drawing of $G$ with a chordless outerface $C_{\text {out }}: v_{1} \rightarrow$ $\ldots v_{k} \rightarrow v_{1}$. We place $v_{1}, \ldots, v_{k}$ in this order along $\ell$ and draw the edges of $C_{o u t}$ as imposed by IP-3. If there is a vertex of $C_{\text {out }}$ with degree less than four, then it is chosen as $v_{k}$ and all invariant properties are satisfied. Otherwise, we appropriately augment our graph, so that IP-4 holds (the detailed proof is given in [1]). We are now ready to state our main theorem.

- Theorem 17. Any planar graph of maximum degree 4 on $n$ vertices admits a two-page book embedding, which can be constructed in $O\left(n^{2}\right)$ time.

Proof. At each step, our algorithm performs a series of computations; the computation of the bridge-blocks, the topological sorting of $G_{a u x}^{T}$, BFS-traversals on the tangency trees. All of these computations can be done in $O(n)$ time, resulting in $O\left(n^{2}\right)$ total time.

\section{Conclusions and Open Problems}

Two approaches were proposed to embed a 4-planar graph into two pages. One reasonable question arising at this point is whether the result can be extended to 5-planar graphs.

\section{References}

1 Michael A. Bekos, Martin Gronemann, and Chrysanthi N. Raftopoulou. Two-page book embeddings of 4-planar graphs. Arxiv report arxiv.org/abs/1401.0684, 2013.

2 Frank Bernhart and Paul C. Kainen. The book thickness of a graph. Journal of Combinatorial Theory, 27(3):320-331, 1979.

3 Chiuyuan Chen. Any maximal planar graph with only one separating triangle is hamiltonian. Journal of Combinatorial Optimization, 7(1):79-86, 2003.

4 Norishige Chiba and Takao Nishizeki. The hamiltonian cycle problem is linear-time solvable for 4-connected planar graphs. Journal of Algorithms, 10(2):187-211, 1989.

5 Fan R. K. Chung, Frank T. Leighton, and Arnold L. Rosenberg. Embedding graphs in books: A layout problem with applications to VLSI design. SIAM Journal on Algebraic and Discrete Methods, 8(1):33-58, 1987.

6 Vida Dujmovic and David R. Wood. On linear layouts of graphs. Discrete Mathematics 83 Theoretical Computer Science, 6(2):339-358, 2004.

7 Lenwood S. Heath. Algorithms for Embedding Graphs in Books. PhD thesis, University of North Carolina, Chapel Hill, 1985.

8 Guido Helden. Each maximal planar graph with exactly two separating triangles is hamiltonian. Discrete Applied Mathematics, 155(14):1833-1836, 2007.

9 Paul C. Kainen and Shannon Overbay. Extension of a theorem of Whitney. Applied Mathematics Letters, 20(7):835-837, 2007.

10 Daniel P. Sanders. On paths in planar graphs. Journal of Graph Theory, 24(4):341-345, 1997.

11 William T. Tutte. A theorem on planar graphs. Transactions of the American Mathematical Society, 82(1):99-116, 1956.

12 Hassler Whitney. A theorem on graphs. Annals of Mathematics, 32(2):378-390, 1931.

13 Avi Wigderson. The complexity of the hamiltonian circuit problem for maximal planar graphs. Technical Report TR-298, EECS Department, Princeton University, 1982.

14 Mihalis Yannakakis. Embedding planar graphs in four pages. Journal of Computer and System Sciences, 38(1):36-67, 1989. 